\title{
ARTICLE \\ Clinical Study \\ Treatment patterns and survival in HER2-positive early breast cancer: a whole-of-population Australian cohort study (2007-2016)
}

\author{
Monica Tang (1] ${ }^{1}$, Andrea Schaffer ${ }^{1}$, Belinda E. Kiely ${ }^{2}$, Benjamin Daniels ${ }^{1}$, Robert J. Simes ${ }^{2}$, Chee K. Lee ${ }^{2}$ and Sallie-Anne Pearson ${ }^{1}$
}

BACKGROUND: Randomised clinical trials (RCTs) demonstrate that trastuzumab improves survival in patients with human epidermal growth factor 2-positive early breast cancer (HER2 + EBC), but real-world patients and clinical practice often differ from RCTs. We examine real-world treatment patterns and outcomes associated with trastuzumab for HER2 + EBC.

METHODS: We identified all Australians dispensed trastuzumab for HER2 + EBC between 1/1/2007 and 30/6/2016. We estimated the proportion of patients completing 12 months of treatment (defined as $\geq 350$ days of exposure within 540 days of initiation). We estimated overall survival (OS) and recurrence-free survival (RFS) by using trastuzumab dispensing for metastatic breast cancer as a surrogate for recurrence.

RESULTS: Our study included 14,644 patients. Among patients with $\geq 540$ days of follow-up $(n=11,903), 67.4 \%$ completed 12 months of trastuzumab. OS rates at 5 and 9 years were 92.7 and $87.9 \%$, and RFS rates at 5 and 9 years were 86.8 and $81.4 \%$, respectively. Patients who completed 12 months of trastuzumab had a 9-year OS rate of $90.2 \%$ compared with $86.2 \%$ among patients receiving $<12$ months of therapy (adjusted HR $0.71,95 \% \mathrm{Cl} 0.62-0.81$ ).

CONCLUSIONS: Real-world HER2 + EBC patients are less likely to complete 12 months of trastuzumab than some clinical trial counterparts but have survival outcomes comparable to those reported in landmark RCTs.

British Journal of Cancer (2019) 121:904-911; https://doi.org/10.1038/s41416-019-0612-5

\section{BACKGROUND}

Human epidermal growth factor 2 (HER2)-positive breast cancers are characterised by amplification of the HER2 gene or overexpression of the HER2 protein and account for $15-20 \%$ of breast malignancies. ${ }^{1}$ The introduction of HER2-targeted therapies has changed the treatment paradigm of HER2-positive cancers, which were previously associated with more aggressive disease and poorer outcomes. ${ }^{2,3}$

Trastuzumab was the first targeted therapy developed to treat HER2-positive breast cancers. Several randomised controlled trials (RCTs) demonstrated that trastuzumab in addition to adjuvant chemotherapy improves disease-free survival (DFS) and overall survival (OS) in women with HER2-positive early breast cancer. ${ }^{4-11}$ The majority of patients in the landmark RCTs received 12 months of adjuvant trastuzumab. ${ }^{4,6,10}$ As subsequent studies investigating the efficacy of shorter durations of adjuvant trastuzumab are conflicting, ${ }^{12-20} 12$ months of trastuzumab remains the standard of care for HER2-positive early breast cancer. As the evidence for prescribing adjuvant trastuzumab is the strongest for high-risk cancers, ${ }^{4,6,10,11}$ not all early breast cancers underwent HER2 testing when trastuzumab was first introduced, ${ }^{21}$ and not all HER2-positive early breast cancers are treated with trastuzumab. ${ }^{21-23}$

The strict eligibility criteria of RCTs limit their generalisability to patients treated in the clinic. Trial participants tend to have fewer adverse events and lower mortality rates than non-participants due to differences in their baseline characteristics and comorbidities. $^{24-28}$ Therefore, data generated from patients treated in routine care are instrumental in assessing whether RCT outcomes translate to the real-world setting. According to previous realworld studies, up to one-third of patients treated with adjuvant trastuzumab do not receive a standard, guideline-recommended chemotherapy regimen, ${ }^{29,30}$ and up to $23 \%$ do not complete the recommended 12-month course of trastuzumab. ${ }^{31-35}$

By virtue of Australia's universal health system, data are available on all patients who accessed trastuzumab for breast cancer since its public subsidy in 2006. In this whole-of-population study, we describe treatment patterns and outcomes associated with adjuvant trastuzumab for early breast cancer. Specifically, we describe patient characteristics, trastuzumab completion rates, survival and cancer recurrence outcomes, and the association of trastuzumab completion with outcomes.

\section{METHODS}

Study setting

In Australia, all citizens and permanent residents have government-subsidised access to prescription medicines listed on the Pharmaceutical Benefits Scheme (PBS). Trastuzumab has

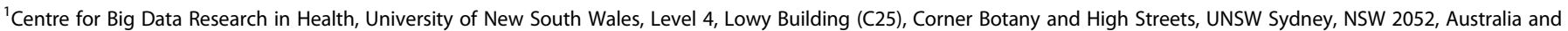
${ }^{2}$ NHMRC Clinical Trials Centre, University of Sydney, Levels 4-6 Medical Foundation Building, 92-94 Parramatta Rd, Camperdown, NSW 2050, Australia Correspondence: Monica Tang (monica.tang@unsw.edu.au)
} 
been PBS-listed for the adjuvant treatment of HER2-positive early breast cancer, irrespective of size and nodal status, since 1 October 2006. Trastuzumab for metastatic breast cancer was funded through the Herceptin Program, outside the PBS, from December 2001 until July 2015, when the Herceptin Program was discontinued and trastuzumab was PBS-listed for all breast cancer stages. We have previously described this whole-ofpopulation cohort of HER2-positive breast cancer patients. ${ }^{36}$ Private insurance in Australia does not reimburse publicly subsidised medicines, so our study population likely captures all Australian residents receiving trastuzumab for HER2-positive early breast cancer.

\section{Data sources}

We conducted a retrospective, population-based, cohort study of all Australians with HER2-positve early breast cancer who received PBS trastuzumab in the adjuvant and neoadjuvant settings. The patient dataset includes sex, year of birth, month/ year of death, remoteness of residence and socioeconomic disadvantage. Fact of death data was supplied in month/year format through linkage to the National Death Index. Cause of death was not available, so it was not possible to estimate breast cancer-specific survival. PBS-dispensing records comprise dispensing date, quantity dispensed and PBS-item number, which identifies the indication for which a medicine is prescribed (e.g. early or metastatic HER2-positive breast cancer) and the specific regimen (e.g. weekly or 3-weekly dosing) (Supplementary Table 1).

\section{Study population}

We included all patients who received $\geq 1$ dispensing of PBSsubsidised trastuzumab for HER2-positive early breast cancer between 1 January 2007 and 30 June 2016. We excluded patients who initiated trastuzumab within the first 3 months of PBS listing of trastuzumab for early breast cancer (1 October-31 December $2006, n=897$ ) as they may have commenced trastuzumab outside the PBS before its public subsidy. We also excluded patients who were dispensed trastuzumab for metastatic HER2-positive breast cancer prior to receiving trastuzumab for early HER2-positive breast cancer $(n=320)$.

\section{Trastuzumab use}

We identified trastuzumab use based on Anatomical Therapeutic Chemical Classification System (ATC) code L01XC03 and PBS-item numbers for early breast cancer.

We assigned 7 or 21 days of exposure to each trastuzumab dispensing based on PBS-item codes for weekly or 3-weekly dosing regimens. We summed the number of days of trastuzumab exposure over all dispensings for each patient. To allow for treatment breaks, we defined completion of a 12-month course of adjuvant trastuzumab as having $\geq 350$ days of trastuzumab exposure within 540 days of trastuzumab initiation. We also examined patients with $\geq 270, \geq 165$ or $\geq 80$ days of trastuzumab exposure. We selected 540 days as the observation time to assess trastuzumab completion to account for patients who may take longer than 12 months to complete a full course of adjuvant trastuzumab due to treatment holidays or concurrent illness; this definition has been used in previous studies of adjuvant trastuzumab using health administrative data. ${ }^{34,35}$ To determine completion rates, we restricted the population to patients with $\geq 540$ days of follow-up (i.e. initiated trastuzumab between 1 January 2007 and 31 December 2014) as this was the observation time used to assess completion of an entire course of adjuvant trastuzumab. We used logistic regression to identify baseline characteristics associated with completion of trastuzumab. We performed sensitivity analyses whereby we defined completion as having $\geq 350$ days of trastuzumab exposure within 365 or 450 days of trastuzumab initiation.
Table 1. Baseline characteristics of study population of all patients dispensed trastuzumab from 2007 to $2016(n=14,644)$

\begin{tabular}{|c|c|c|}
\hline & Frequency & (\%) \\
\hline \multicolumn{3}{|c|}{ Age at the first trastuzumab dispensing } \\
\hline$\leq 30$ years & 179 & 1.2 \\
\hline $31-40$ years & 1354 & 9.3 \\
\hline $41-50$ years & 3661 & 25 \\
\hline $51-60$ years & 4558 & 31.1 \\
\hline $61-70$ years & 3333 & 22.8 \\
\hline $71-80$ years & 1349 & 9.2 \\
\hline$>80$ years & 210 & 1.4 \\
\hline \multicolumn{3}{|l|}{ Sex } \\
\hline Female & 14,590 & 99.6 \\
\hline Male & 54 & 0.4 \\
\hline \multicolumn{3}{|c|}{ Year of the first trastuzumab dispensing } \\
\hline 2007-2008 & 2353 & 16.1 \\
\hline 2009-2010 & 2823 & 19.3 \\
\hline 2011-2012 & 3068 & 21 \\
\hline 2013-2014 & 3659 & 25 \\
\hline 2015-2016 & 2741 & 18.7 \\
\hline \multicolumn{3}{|l|}{ Remoteness } \\
\hline Capital cities & 9177 & 62.7 \\
\hline Other metropolitan centres & 1155 & 7.9 \\
\hline Large rural centres & 965 & 6.6 \\
\hline Other rural & 2984 & 20.4 \\
\hline Remote & 238 & 1.6 \\
\hline Missing & 125 & 0.9 \\
\hline \multicolumn{3}{|l|}{ State } \\
\hline New South Wales & 4892 & 33.4 \\
\hline Australian Capital Territory & 210 & 1.4 \\
\hline Northern Territory & 122 & 0.8 \\
\hline Queensland & 2890 & 19.7 \\
\hline South Australia & 1098 & 7.5 \\
\hline Tasmania & 304 & 2.1 \\
\hline Victoria & 3864 & 26.4 \\
\hline Western Australia & 1264 & 8.6 \\
\hline \multicolumn{3}{|c|}{ Index of Relative Socioeconomic Disadvantage } \\
\hline 1 (most disadvantage) & 1862 & 12.7 \\
\hline 2 & 3201 & 21.9 \\
\hline 3 & 2873 & 19.6 \\
\hline 4 & 3413 & 23.3 \\
\hline 5 (least disadvantage) & 3234 & 22.1 \\
\hline Missing & 61 & 0.4 \\
\hline
\end{tabular}

Concomitant chemotherapy and endocrine therapy

We determined commonly prescribed (neo)adjuvant chemotherapy regimens based on the dispensing of cytotoxic agents, identified by ATC codes commencing with L01, in the period 120 days before until 60 days after the first trastuzumab dispensing. We defined oestrogen receptor (ER)-positive patients as those who were dispensed endocrine therapy within 120 days before or 540 days after the first trastuzumab dispensing. ${ }^{37}$ We identified patients who received a selective oestrogen receptor modulator (tamoxifen or toremifene) and/or aromatase inhibitor (anastrozole, letrozole or exemestane) by using ATC codes. 
Table 2. Baseline factors associated with completion of treatment (logistic regression) in patients with at least 540 days of follow-up from the first trastuzumab dispensing (i.e. initiated trastuzumab 2007-2014, $n=11903$ )

\begin{tabular}{|c|c|c|c|c|c|c|}
\hline & Completed the course & Did not complete the course & \multicolumn{2}{|c|}{ Univariate analysis } & \multicolumn{2}{|c|}{ Multivariable analysis } \\
\hline \multicolumn{7}{|l|}{ Age category } \\
\hline$\leq 30$ years & 102 & 46 & 1 & Reference & 1 & \\
\hline $31-40$ years & 757 & 355 & 0.96 & $0.66-1.39$ & 0.96 & $0.66-1.40$ \\
\hline $41-50$ years & 2068 & 942 & 0.99 & $0.69-1.41$ & 1.02 & $0.71-1.46$ \\
\hline $71-80$ years & 646 & 372 & 0.78 & $0.54-1.14$ & 0.77 & $0.53-1.12$ \\
\hline $80+$ years & 74 & 73 & 0.46 & $0.28-0.74$ & 0.42 & $0.26-0.68$ \\
\hline \multicolumn{7}{|l|}{ State } \\
\hline New South Wales & 2764 & 1185 & 1 & & 1 & \\
\hline Australian Capital Territory & 98 & 66 & 0.64 & $0.46-0.87$ & 0.53 & $0.38-0.74$ \\
\hline Tasmania & 152 & 89 & 0.73 & $0.56-0.96$ & 0.75 & $0.57-0.99$ \\
\hline Victoria & 2231 & 936 & 1.02 & $0.92-1.13$ & 1.02 & $0.91-1.13$ \\
\hline Western Australia & 674 & 346 & 0.84 & $0.72-0.97$ & 0.81 & $0.69-0.94$ \\
\hline \multicolumn{7}{|c|}{ Index of relative socioeconomic disadvantage } \\
\hline 1 (most disadvantage) & 946 & 529 & 1 & & 1 & \\
\hline 2 & 1711 & 905 & 1.06 & $0.93-1.21$ & 1.05 & $0.92-1.21$ \\
\hline 3 & 1549 & 797 & 1.09 & $0.95-1.25$ & 1.03 & $0.89-1.19$ \\
\hline 4 & 1921 & 879 & 1.22 & $1.07-1.40$ & 1.13 & $0.98-1.29$ \\
\hline 5 (least disadvantage) & 1869 & 761 & 1.37 & $1.20-1.57$ & 1.23 & $1.06-1.42$ \\
\hline Missing & 22 & 14 & 0.88 & $0.45-1.73$ & 0.74 & $0.36-1.51$ \\
\hline \multicolumn{7}{|c|}{ Year of the first trastuzumab dispensing } \\
\hline 2007-2008 & 1427 & 926 & 1 & & 1 & \\
\hline 2009-2010 & 1872 & 951 & 1.28 & $1.14-1.43$ & 1.28 & $1.14-1.44$ \\
\hline 2011-2012 & 2147 & 921 & 1.51 & $1.35-1.69$ & 1.55 & $1.38-1.74$ \\
\hline 2013-2014 & 2572 & 1087 & 1.54 & $1.38-1.71$ & 1.62 & $1.45-1.81$ \\
\hline
\end{tabular}

\section{Outcomes}

We calculated OS as the time between the first dispensing of adjuvant trastuzumab to the date of death, set as the last day of each month, as fact of death data was supplied in month and year format. All individuals had follow-up for date of death data until 31 May 2017. We used Kaplan-Meier curves and Cox regression analysis to examine factors associated with OS, including baseline characteristics and completion of trastuzumab course. When examining associations between trastuzumab completion and OS, we excluded patients who died within 540 days of trastuzumab initiation and did not have the opportunity to receive a full 12-month course. ${ }^{38}$

We used the date of the first trastuzumab dispensing for metastatic disease as a surrogate for recurrence of HER2positive breast cancer, based on PBS-dispensing records available until 30 June 2016. We defined time to recurrence as the time between the first dispensing of adjuvant trastuzumab to the first trastuzumab dispensing for metastatic disease. We calculated recurrence-free survival (RFS) from the date of the first adjuvant trastuzumab dispensing to the date of recurrence or death.

For patients with at least 5 years of dispensing data follow-up (i.e. the first trastuzumab dispensing between 1 January 2007 and 30 June 2011), we calculated the number of recurrences occurring in each year of follow-up.

Ethics and data access

This study was approved by the NSW Population and Health Services Research Ethics Committee (Approval Number: 2010/02/ 213). The Australian Department of Human Services (DHS) External Request Evaluation Committee approved access to the data (Approval Numbers: MI1474, MI1475, MI1477 and MI5858). 


\section{RESULTS}

Study population

Between 1 October 2006 and 30 June 2016, 15,861 Australians were dispensed at least one dose of (neo)adjuvant trastuzumab for HER2-positive early breast cancer. Of these, 14,644 patients received their first trastuzumab dispensing between 1 January 2007 and 30 June 2016 and were included in our study (Table 1). Median age at the first trastuzumab dispensing was 55 years (range 22-94, interquartile range [IQR] 47-74). Most of them resided in a capital city $(62.7 \%)$, and patients from the quintile with the most socioeconomic disadvantage were underrepresented (12.7\%)

Trastuzumab use

Patients initiating adjuvant trastuzumab between 1 January 2007 and 30 June $2016(n=14,644)$ had a median of 17 trastuzumab dispensings (IQR 15-18).

Among patients with at least 540 days of follow-up $(n=11,903)$, $8018(67.4 \%)$ completed 12 months of adjuvant trastuzumab (i.e.

Table 3. Chemotherapy regimens based on dispensings between 120 days before and 60 days after trastuzumab initiation $(n=14,644)$

\begin{tabular}{lcc}
\hline & Frequency & $(\%)$ \\
\hline No chemotherapy drugs dispensed & 565 & 4.9 \\
At least one chemotherapy drug dispensed & 14,079 & 96.1 \\
Anthracycline- and taxane-based & 6809 & 46.5 \\
AC-TH & 3287 & 22.4 \\
FEC-DH & 1561 & 13.7 \\
Other & 1961 & 13.4 \\
Taxane-based (no anthracycline) & 6664 & 45.5 \\
TCH & 3293 & 22.5 \\
Paclitaxel-trastuzumab & 1517 & 10.4 \\
Docetaxel-trastuzumab & 586 & 4 \\
Other & & \\
Anthracycline-based (no taxane) & 426 & 2.9 \\
No anthracycline or taxane & 180 & 1.2 \\
CMF-H & 25 & 0.2 \\
Other & 155 & 1.1 \\
\hline AC-TH doxorubicin, cyclophosphamide, taxane and trastuzumab, FEC-DH 5- \\
fluorouracil, epirubicin, cyclophosphamide, docetaxel and trastuzumab, \\
TCH docetaxel, carboplatin and trastuzumab, CMF-H cyclophosphamide, \\
methotrexate, 5-fluorouracil and trastuzumab \\
\hline
\end{tabular}

were dispensed $\geq 350$ days of trastuzumab). $21.9 \%$ received 270-349 days of therapy, $5.6 \%$ received $165-269$ days, $3.3 \%$ received $80-164$ days and $1.8 \%$ received $<80$ days. Age $>80$, socioeconomic disadvantage and residing outside metropolitan areas were associated with lower likelihood of completing trastuzumab (Table 2). Patients initiating trastuzumab in later years were more likely to complete a 12-month course compared with those initiating in 2007-2008. In our sensitivity analyses, $58.0 \%$ of patients completed 12 months of trastuzumab within 365 days of initiation, and $66.3 \%$ of patients completed 12 months of trastuzumab within 450 days of initiation.

\section{Chemotherapy}

Among patients initiating adjuvant trastuzumab between 1 January 2007 and 30 June 2016, 14,079 (96.1\%) were dispensed at least one dose of concomitant chemotherapy (Table 3). Of these, nearly all received a taxane-based regimen, with $(48.4 \%)$ or without an anthracycline (47.3\%). Patients dispensed anthracyclines and taxanes were younger than those receiving nonanthracycline taxane-based regimens (mean 52.6 vs. 57.9 years). Among patients with at least 540 days of follow-up, we did not observe an association between receiving anthracycline and likelihood of completing 12 months of trastuzumab (HR 0.95 for anthracycline- and taxane-based regimens vs. non-anthracycline taxane-based regimens, $95 \% \mathrm{Cl} 0.87-1.04$ ).

Endocrine therapy

More than half of patients $(n=8,444,57.7 \%)$ received at least one dispensing of endocrine therapy. The mean ages of endocrine therapy recipients and non-recipients were similar (54.8 vs. 55.9 years).

\section{Outcomes}

Overall survival. By 31 May 2017, there were 1044 deaths (7.1\%) among 14,644 patients initiating trastuzumab between 1 January 2007 and 30 June 2016, after median follow-up time of 4.7 years. The annual OS probabilities for this cohort are presented in Fig. 1.

In a multivariable model examining baseline characteristics, older age at the first trastuzumab dispensing, socioeconomic disadvantage and earlier year of trastuzumab initiation were associated with poorer survival (Table 4).

Survival by completion of trastuzumab course. In our multivariable analysis, excluding 133 patients who died within 540 days of their first dispensing of trastuzumab, not completing 12 months of adjuvant trastuzumab was associated with shorter OS (HR 1.41, $95 \% \mathrm{Cl}$ 1.23-1.62), after adjusting for age, socioeconomic disadvantage and year of trastuzumab initiation. Five- and

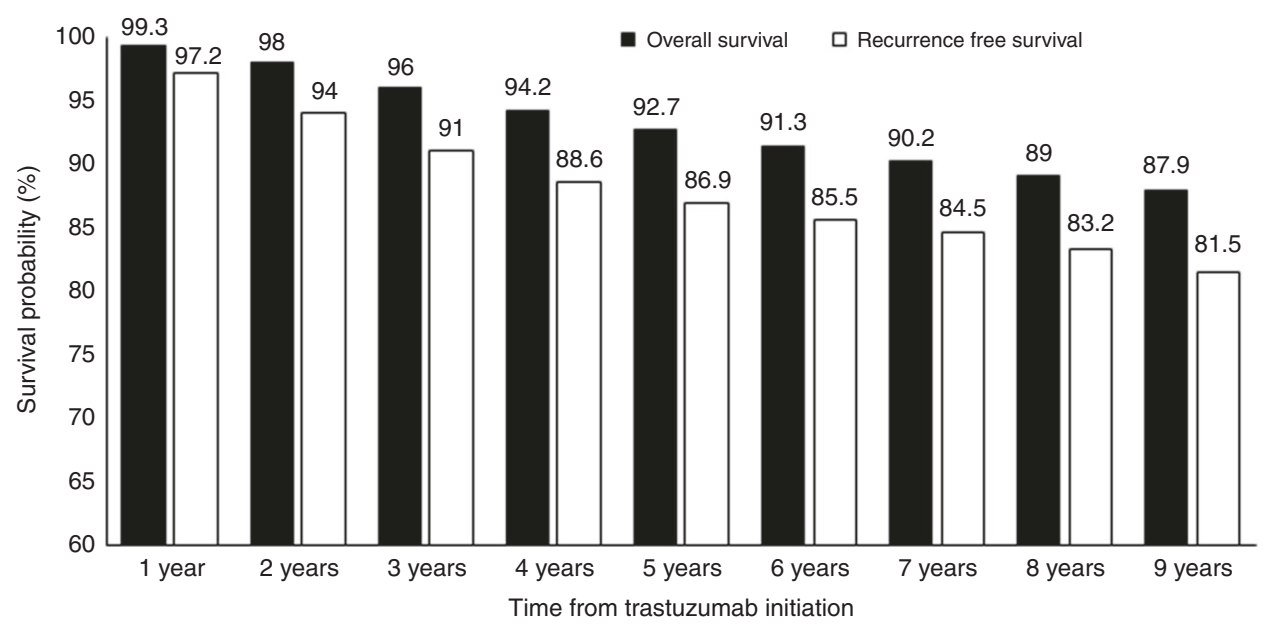

Fig. 1 Annual overall and recurrence-free survival probabilities $(n=14644)$ of patients still in follow-up 
Table 4. Baseline characteristics associated with overall survival $(n=14644)$

\begin{tabular}{|c|c|c|c|c|}
\hline & \multicolumn{2}{|c|}{ Univariate analysis } & \multicolumn{2}{|c|}{ Multivariable analysis } \\
\hline & Hazard ratio & $95 \% \mathrm{Cl}$ & Hazard ratio & $95 \% \mathrm{Cl}$ \\
\hline \multicolumn{5}{|l|}{ Age category } \\
\hline$\leq 30$ years & 1 & & 1 & \\
\hline $31-40$ years & 0.81 & $0.45-1.46$ & 0.81 & $0.45-1.46$ \\
\hline $41-50$ years & 0.69 & $0.39-1.21$ & 0.68 & $0.39-1.19$ \\
\hline $51-60$ years & 0.79 & $0.45-1.37$ & 0.76 & $0.44-1.33$ \\
\hline $61-70$ years & 1.16 & $0.67-2.03$ & 0.13 & $0.65-1.98$ \\
\hline $71-80$ years & 2.05 & $1.17-3.61$ & 2.07 & $1.17-3.63$ \\
\hline $80+$ years & 6.11 & $3.35-11.14$ & 6.45 & $3.53-11.78$ \\
\hline \multicolumn{5}{|l|}{ Remoteness } \\
\hline Capital cities & 1 & & & \\
\hline $\begin{array}{l}\text { Other } \\
\text { metropolitan } \\
\text { centres }\end{array}$ & 1.04 & $0.82-1.32$ & & \\
\hline $\begin{array}{l}\text { Large rural } \\
\text { centres }\end{array}$ & 1.11 & $0.87-1.41$ & & \\
\hline Other rural & 1.15 & $0.99-1.33$ & & \\
\hline Remote & 0.92 & $0.54-1.57$ & & \\
\hline Missing & 1.32 & $0.73-2.40$ & & \\
\hline \multicolumn{5}{|l|}{ State } \\
\hline $\begin{array}{l}\text { New } \\
\text { South Wales }\end{array}$ & 1 & & & \\
\hline $\begin{array}{l}\text { Australian } \\
\text { Capital } \\
\text { Territory }\end{array}$ & 1.23 & $0.76-2.01$ & & \\
\hline $\begin{array}{l}\text { Northern } \\
\text { Territory }\end{array}$ & 0.63 & $0.28-1.42$ & & \\
\hline Queensland & 0.9 & $0.76-1.07$ & & \\
\hline South Australia & 1.08 & $0.86-1.36$ & & \\
\hline Tasmania & 0.96 & $0.60-1.55$ & & \\
\hline Victoria & 0.97 & $0.83-1.14$ & & \\
\hline $\begin{array}{l}\text { Western } \\
\text { Australia }\end{array}$ & 0.76 & $0.58-0.98$ & & \\
\hline \multicolumn{5}{|c|}{ Index of relative socioeconomic disadvantage } \\
\hline $\begin{array}{l}1 \text { (most } \\
\text { disadvantage) }\end{array}$ & 1 & & 1 & \\
\hline 2 & 0.97 & $0.08-1.19$ & 0.95 & $0.78-1.15$ \\
\hline 3 & 0.78 & $0.63-0.97$ & 0.76 & $0.62-0.94$ \\
\hline 4 & 0.8 & $0.66-0.98$ & 0.78 & $0.64-0.96$ \\
\hline $\begin{array}{l}5 \text { (least } \\
\text { disadvantage) }\end{array}$ & 0.63 & $0.51-0.78$ & 0.6 & $0.48-0.74$ \\
\hline Missing & 1.56 & $0.64-3.80$ & 1.75 & $0.72-4.27$ \\
\hline \multicolumn{5}{|c|}{ Year of the first dispensing } \\
\hline $2007-2008$ & 1 & & 1 & \\
\hline 2009-2010 & 1.04 & $0.88-1.23$ & 0.99 & $0.84-1.16$ \\
\hline 2011-2012 & 0.86 & $0.71-1.03$ & 0.79 & $0.66-0.95$ \\
\hline 2013-2014 & 0.91 & $0.74-1.12$ & 0.78 & $0.63-0.95$ \\
\hline 2015-2016 & 0.91 & $0.64-1.29$ & 0.74 & $0.52-1.05$ \\
\hline
\end{tabular}

9-year survival probabilities were 94.6 and $90.2 \%$ among patients who completed 12 months of trastuzumab, and 91.8 and $86.2 \%$ among those who did not.

Recurrence. By 30 June 2016, 1,027 patients (7.0\%) received at least one dispensing of trastuzumab for metastatic HER2-positive breast cancer. Figure 1 describes the annual RFS rates for patients commencing trastuzumab between 2007 and 2016.

Among patients with at least 5 years of follow-up $(n=5,926)$, 526 had a cancer recurrence (8.8\%). Median duration of follow-up was 6.9 years (IQR 5.7-8.1 years). The number of recurrences in each year of follow-up is described in Fig. 2.

\section{DISCUSSION}

We report on nearly 15,000 patients who received trastuzumab for non-metastatic breast cancer in Australia between 2007 and 2016; to our knowledge, this is the largest cohort of HER2-positive breast cancer patients studied in a population-based setting. Survival outcomes in this observational study are similar to those reported in the landmark RCTs in HER2-positive early breast cancer., $4,6,10$ However, in contrast to some clinical trials, only two-thirds of patients initiating trastuzumab in our study completed the standard 12-month course.

This study illustrates the differences between RCT populations and patients treated in clinical practice and highlights the value of real-world studies in verifying and complementing clinical trial findings. The proportion of patients aged $\geq 60$ years is twice as high in our study (33\%) as in seminal RCTs of adjuvant trastuzumab $(16 \%),{ }^{4,6}$ confirming underrepresentation of older patients in clinical trials. ${ }^{25,26}$ The 12 -month trastuzumab completion rate of $67.2 \%$ in our study was similar to the treatment completion rate of $68.6 \%$ in the NCCTG N9831/NSABP B-31 trials, ${ }^{4}$ but lower than $91.5 \%$ reported in the HERA trial. ${ }^{6}$ In our study, patients who did not complete 12 months of trastuzumab were more likely to be older, be socioeconomically disadvantaged and reside in rural and remote areas, all groups underrepresented in clinical trials. ${ }^{27,39,40}$

Despite lower completion rates, the outcomes of our study are comparable to landmark clinical trials; the 5-year OS rate in our study is $92.7 \%$, compared with the 4 -year OS rate of $89.3 \%$ in the HERA trial ${ }^{8}$ and 5 -year OS rates of $91-92 \%$ in the BCIRG 006 trial. $^{10}$ Our 9-year OS rate $(87.9 \%)$ was similar to the 10 -year OS $(84 \%)$ reported in the NCCTG N98931/NSABP B-31 trials. ${ }^{5}$ OS estimates in our study are probably ameliorated by the absence of requirements for minimum tumour size or nodal status for accessing trastuzumab for HER2-positive early breast cancer in Australia, whereas most RCTs recruited only patients with tumours that were lymph node positive and/or larger than 1-2 cm. 4,6,10 Therefore, we hypothesise that patients in our study were more likely to have cancers with favourable prognostic features than those in clinical trials, a finding that has been reported in previous real-world studies. ${ }^{35,41,42}$ The introduction of novel HER2-targeted agents for patients with recurrent metastatic disease probably also improved OS rates in our study, particularly for patients diagnosed in later years.

Patients who did not complete 1 year of trastuzumab had a $41 \%$ increased risk of death compared with those who did (HR 1.41), although the absolute difference between the groups is small (9year OS rate 86.2 vs. $90.2 \%$ ). The difference in survival associated with completing a year of trastuzumab in our study is larger than that reported in a meta-analysis of RCTs comparing 12 months with shorter durations of trastuzumab (HR 1.16). ${ }^{43}$ This is probably due to differences between clinical trial and real-world populations, as well as unmeasured confounders in our study, such as baseline cardiac impairment, that increase the likelihood of both early treatment cessation and death.

We report 4- and 5-year RFS rates of 88.4 and $86.8 \%$, respectively. These figures may underestimate true recurrence rates as they are based on the receipt of trastuzumab for metastatic disease as a surrogate for recurrence. However, they are not dissimilar to 4-year DFS rates of $78.6-85.3 \%$ and 5-year DFS rates of $81-84 \%$ reported in clinical trials. $8,10,44$ Among patients with $\geq 5$ years of follow-up, $87 \%$ of all recurrences occurred within 5 years of initiating adjuvant trastuzumab (Fig. 2). The majority of 


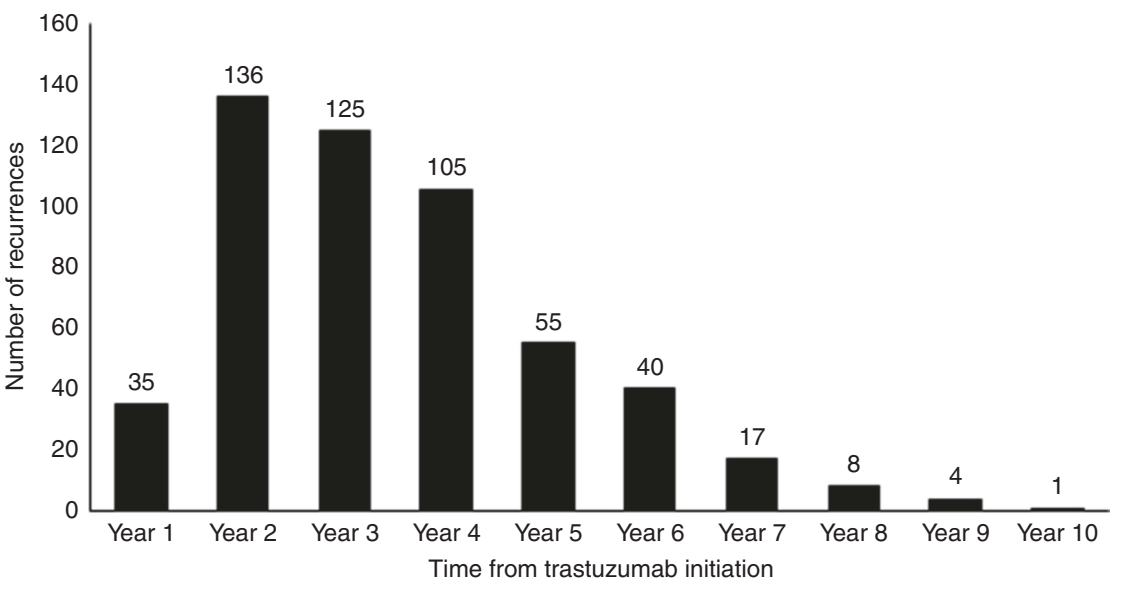

Fig. 2 Number of recurrences by year of follow-up for patients with at least 5 years of follow-up $(n=5926)$

patients $(70 \%)$ who started trastuzumab for metastatic disease did so during years 2-4 after their first dose of adjuvant trastuzumab. This suggests that the first 3 years after completing adjuvant trastuzumab are the most clinically relevant time period for monitoring and detecting recurrent metastatic disease in HER2positive breast cancer.

When novel treatments are considered for public reimbursement, policy makers need reliable data on estimated treatment uptake and efficacy to support decision-making. Therefore, lowerthan-expected rates of trastuzumab completion in routine practice have implications for the real-world applicability and assessment for reimbursement of novel HER2-targeted agents, such as neratinib, that are intended for use after patients complete 12 months of adjuvant trastuzumab. ${ }^{45}$ Patients in remote regions were $53 \%$ less likely to complete treatment compared with patients in capital cities, and those with the least socioeconomic disadvantage were $23 \%$ more likely to complete treatment than those with the most disadvantage. Therefore, geographical and financial barriers may limit patients' ability to complete recommended treatment and potentially compromise cancer outcomes, although remoteness was not associated with survival in our study.

The major strength of our study lies in our ability to use population-wide medicines dispensing and survival data from a national, representative, cohort across all age groups, not just over $65 \mathrm{~s}$. However, the trade-off in utilising population-wide health administrative data is the lack of detailed clinical information, which contrasts with studies that have this information but are most commonly conducted in smaller and not necessarily generalisable populations. Our study is limited by the lack of clinical details such as tumour size and stage and patient comorbidities, including pre-existing cardiac disease. We did not have information on cause of death and therefore examined OS only. We also did not have access to clinical records documenting reasons for non-completion of 12 months of trastuzumab or exact dates or sites of relapse of metastatic disease.

\section{CONCLUSION}

Although HER2-positive early breast cancer patients in the community are less likely to complete a 12-month course of adjuvant trastuzumab than clinical trial counterparts, survival and recurrence rates in this real-world population are reassuringly comparable to those reported in landmark clinical trials. Realworld studies of cancer medicine utilisation and outcomes provide valuable data to substantiate clinical trial results and support clinical and policy decision-making.

\section{AUTHOR CONTRIBUTIONS}

M.T., A.S. and S.P. were responsible for conceiving of and designing the study. M.T. and A.S. were responsible for statistical analysis. M.T., A.S., B.E.K., B.D., R.J.S., C.K.L. and S.P. were responsible for data analysis and interpretation. M.T. was responsible for writing the paper. M.T., A.S., B.E.K., B.D., R.J.S., C.K.L. and S.P. were responsible for editing and reviewing the paper.

\section{ADDITIONAL INFORMATION}

Supplementary information is available for this paper at https://doi.org/10.1038/ s41416-019-0612-5.

Competing interests: M.T. reports travel support from Roche, outside the submitted work. B.E.K. reports funding from Roche and TEVA for participating in advisory boards; speakers fees from Roche and Novartis; travel/conference expenses from Roche. S.A.P. is a member of the Drug Utilisation Sub Committee of the Pharmaceutical Benefits Advisory Committee. The views expressed in this paper do not represent those of the committee. The remaining authors declare no competing interests.

Ethics approval and consent to participate: This study was approved by the New South Wales Population and Health Services Research Ethics Committee (Approval Number: 2010/02/213). The Ethics Committee granted a waiver of the usual requirement for the consent of the individual to the use of their health information in a research project, in line with the guidelines approved under Section 95/95A of the Privacy Act 1988. The study was performed in accordance with the Declaration of Helsinki.

Funding: This research is supported by the National Health and Medical Research Council (NHMRC) Centre of Research Excellence in Medicines and Ageing (ID: 1060407) and a Cooperative Research Centre Project (CRC-P) Grant from the Australian Government Department of Industry, Innovation and Science (ID: CRC-P439). M.T. is supported by a NHMRC Postgraduate Research Scholarship (ID: 1151479), a National Breast Cancer Foundation Postgraduate Scholarship Top-Up (ID: DS-18-01), and a Translational Cancer Research Network Clinical PhD Scholarship Top-Up award, supported by the Cancer Institute NSW. A.S. is supported by a NHMRC Early Career Fellowship (ID: 1158763).

Consent to publish: Not applicable.

Data availability: We thank the Australian Government Department of Human Services for providing the data. Data access was granted by the Australian Government Department of Human Services External Request Evaluation Committee (Approval Numbers: MI1474, MI1475, MI1477 and MI5858). Access to the datasets analysed during this study is not permitted without the express permission of the approving human research ethics committees and data custodians.

Note: This work is published under the standard license to publish agreement. After 12 months the work will become freely available and the license terms will switch to a Creative Commons Attribution 4.0 International (CC BY 4.0). 
Publisher's note Springer Nature remains neutral with regard to jurisdictional claims in published maps and institutional affiliations.

\section{REFERENCES}

1. Wolff, A. C., Hammond, M. E., Hicks, D. G., Dowsett, M., McShane, L. M., Allison, K. $\mathrm{H}$. et al. Recommendations for human epidermal growth factor receptor 2 testing in breast cancer: American Society of Clinical Oncology/College of American Pathologists clinical practice guideline update. J. Clin. Oncol. 31, 3997-4013 (2013).

2. Slamon, D. J., Clark, G. M., Wong, S. G., Levin, W. J., Ullrich, A. \& McGuire, W. L. Human breast cancer: correlation of relapse and survival with amplification of the HER-2/neu oncogene. Science 235, 177-182 (1987).

3. Loibl, S. \& Gianni, L. HER2-positive breast cancer. Lancet 389, 2415-2429 (2017).

4. Romond, E. H., Perez, E. A., Bryant, J., Suman, V. J., Geyer, C. E., Davidson, N. E. et al. Trastuzumab plus adjuvant chemotherapy for operable HER2-positive breast cancer. N. Engl. J. Med. 353, 1673-1684 (2005).

5. Perez, E. A., Romond, E. H., Suman, V. J., Jeong, J.-H., Sledge, G., Geyer, C. E. Jr et al. Trastuzumab plus adjuvant chemotherapy for human epidermal growth factor receptor 2-positive breast cancer: planned joint analysis of overall survival from NSABP B-31 and NCCTG N9831. J. Clin. Oncol. 32, 3744 (2014).

6. Piccart-Gebhart, M. J., Procter, M., Leyland-Jones, B., Goldhirsch, A., Untch, M., Smith, I. et al. Trastuzumab after adjuvant chemotherapy in HER2-positive breast cancer. N. Engl. J. Med. 353, 1659-1672 (2005).

7. Smith, I., Procter, M., Gelber, R. D., Guillaume, S., Feyereislova, A., Dowsett, M. et al. 2-year follow-up of trastuzumab after adjuvant chemotherapy in HER2-positive breast cancer: a randomised controlled trial. Lancet 369, 29-36 (2007).

8. Gianni, L., Dafni, U., Gelber, R. D., Azambuja, E., Muehlbauer, S., Goldhirsch, A. et al. Treatment with trastuzumab for 1 year after adjuvant chemotherapy in patients with HER2-positive early breast cancer: a 4-year follow-up of a randomised controlled trial. Lancet Oncol. 12, 236-244 (2011).

9. Goldhirsch, A., Gelber, R. D., Piccart-Gebhart, M. J., de Azambuja, E., Procter, M., Suter, T. M. et al. 2 years versus 1 year of adjuvant trastuzumab for HER2-positive breast cancer (HERA): an open-label, randomised controlled trial. Lancet 382, 1021-1028 (2013).

10. Slamon, D., Eiermann, W., Robert, N., Pienkowski, T., Martin, M., Press, M. et al. Adjuvant trastuzumab in HER2-positive breast cancer. N. Engl. J. Med. 365, 1273-1283 (2011).

11. Joensuu, H., Kellokumpu-Lehtinen, P.-L., Bono, P., Alanko, T., Kataja, V., Asola, R. et al. Adjuvant docetaxel or vinorelbine with or without trastuzumab for breast cancer. N. Engl. J. Med. 354, 809-820 (2006).

12. Pivot, X., Romieu, G., Debled, M., Pierga, J. Y., Kerbrat, P., Bachelot, T. et al. 6 months versus 12 months of adjuvant trastuzumab for patients with HER2positive early breast cancer (PHARE): a randomised phase 3 trial. Lancet Oncol. 14, 741-748 (2013).

13. Pivot, X., Romieu, G., Debled, M., Pierga, J.-Y., Kerbrat, P., Bachelot, T. et al. 6 months versus 12 months of adjuvant trastuzumab in early breast cancer (PHARE): final analysis of a multicentre, open-label, phase 3 randomised trial. Lancet 393, 2591-2598 (2019).

14. Mavroudis, D., Saloustros, E., Malamos, N., Kakolyris, S., Boukovinas, I., Papakotoulas, P. et al. Six versus 12 months of adjuvant trastuzumab in combination with dose-dense chemotherapy for women with HER2-positive breast cancer: a multicenter randomized study by the Hellenic Oncology Research Group (HORG). Ann. Oncol. 26, 1333-1340 (2015).

15. Conte, P., Frassoldati, A., Bisagni, G., Brandes, A. A., Donadio, M., Garrone, O. et al. Nine weeks vs 1 year adjuvant trastuzumab in combination with chemotherapy: final results of the phase III randomized Short-HER study. Ann. Oncol. 29, 2328-2333 (2018).

16. Earl, H. M., Hiller, L., Vallier, A.-L., Loi, S., McAdam, K., Hughes-Davies, L. et al. 6 versus 12 months of adjuvant trastuzumab for HER2-positive early breast cancer (PERSEPHONE): 4-year disease-free survival results of a randomised phase 3 noninferiority trial. The Lancet. 393, 2599-2612. https://doi.org/10.1016/S0140-6736 (19)30650-6. Epub 2019 Jun 6.

17. Earl, H. M., Hiller, L., Vallier, A.-L., Loi, S., McAdam, K., Hughes-Davies, L. et al. 6 versus 12 months of adjuvant trastuzumab for HER2-positive early breast cancer (PERSEPHONE): 4-year disease-free survival results of a randomised phase 3 noninferiority trial. Lancet 393, 2599-2612 (2019).

18. Joensuu, H., Fraser, J., Wildiers, H. et al. Effect of adjuvant trastuzumab for a duration of 9 weeks vs 1 year with concomitant chemotherapy for early human epidermal growth factor receptor 2-positive breast cancer: The sold randomized clinical trial. JAMA Oncol. 4, 1199-1206 (2018).

19. Senkus, E., Kyriakides, S., Ohno, S., Penault-Llorca, F., Poortmans, P., Rutgers, E. et al. Primary breast cancer: ESMO Clinical Practice Guidelines for diagnosis, treatment and follow-upt. Ann. Oncol. 26(suppl_5), v8-v30 (2015).
20. National Comprehensive Cancer Network. NCCN Guidelines Breast Cancer Version 1. In: National ComprehensiveCancer Netowrk; 2019.

21. Whitfield, R., Kollias, J., De Silva, P., Zorbas, H. \& Maddern, G. Use of trastuzumab in Australia and New Zealand: results from the National Breast Cancer Audit. ANZ J. Surg. 82, 234-239 (2012).

22. Pagani, O., Regan, M. M., Walley, B. A., Fleming, G. F., Colleoni, M., Láng, I. et al. Adjuvant exemestane with ovarian suppression in premenopausal breast cancer. N. Engl. J. Med. 371, 107-118 (2014).

23. Harris, C. A., Pearson, S., Daniels, B., Srasuebkul, P. \& Ward, R. L. HER2-positive early breast cancer (HER2EBC): an Australian pattern of care study. J. Clin. Oncol. 31, 101-101 (2013).

24. Avorn, J. In defense of pharmacoepidemiology-embracing the yin and yang of drug research. N. Engl. J. Med. 357, 2219 (2007).

25. Hutchins, L. F., Unger, J. M., Crowley, J. J., Coltman, C. A. Jr. \& Albain, K. S. Underrepresentation of patients 65 years of age or older in cancer-treatment trials. N. Engl. J. Med. 341, 2061-2067 (1999).

26. Lewis, J. H., Kilgore, M. L., Goldman, D. P., Trimble, E. L., Kaplan, R., Montello, M. J. et al. Participation of patients 65 years of age or older in cancer clinical trials. J. Clin.Oncol. 21, 1383-1389 (2003).

27. Van Spall, H. G., Toren, A., Kiss, A. \& Fowler, R. A. Eligibility criteria of randomized controlled trials published in high-impact general medical journals: a systematic sampling review. JAMA 297, 1233-1240 (2007).

28. Davis, S., Wright, P. W., Schulman, S. F., Hill, L. D., Pinkham, R. D., Johnson, L. P. et al. Participants in prospective, randomized clinical trials for resected non-small cell lung cancer have improved survival compared with nonparticipants in such trials. Cancer 56, 1710-1718 (1985).

29. Freedman, R. A., Vaz-Luis, I., Barry, W. T., Lii, H., Lin, N. U., Winer, E. P. et al. Patterns of chemotherapy, toxicity, and short-term outcomes for older women receiving adjuvant trastuzumab-based therapy. Breast Cancer Res Treat 145, 491-501 (2014).

30. Reeder-Hayes, K., Peacock Hinton, S., Meng, K., Carey, L. A. \& Dusetzina, S. B. Disparities in Use of Human Epidermal Growth Hormone Receptor 2-Targeted Therapy for Early-Stage Breast Cancer. J. Clin. Oncol. 34, 2003-2009 (2016).

31. Chan, A. \& McGregor, S. R. Prevalence and management of HER2/neu-positive early breast cancer in a single institution following availability of adjuvant trastuzumab. Intern. Med. J. 42, 267-274 (2012).

32. Campiglio, M., Bufalino, R., Sasso, M., Ferri, E., Casalini, P., Adamo, V. et al. Effect of adjuvant trastuzumab treatment in conventional clinical setting: an observational retrospective multicenter Italian study. Breast Cancer Res. Treat. 141, 101-110 (2013).

33. Freedman, R. A., Hughes, M. E., Ottesen, R. A., Weeks, J. C., He, Y., Wong, Y. N. et al. Use of adjuvant trastuzumab in women with human epidermal growth factor receptor 2 (HER2)-positive breast cancer by race/ethnicity and education within the National Comprehensive Cancer Network. Cancer 119, 839-846 (2013).

34. Vaz-Luis, I., Keating, N. L., Lin, N. U., Lii, H., Winer, E. P. \& Freedman, R. A. Duration and toxicity of adjuvant trastuzumab in older patients with early-stage breast cancer: a population-based study. J. Clin. Oncol. 32, 927-934 (2014).

35. Reeder-Hayes, K. E., Meyer, A. M., Hinton, S. P., Meng, K., Carey, L. A. \& Dusetzina, S. B. Comparative toxicity and effectiveness of trastuzumab-based chemotherapy regimens in older women with early-stage breast cancer. J. Clin. Oncol. 35, 3298-3305 (2017).

36. Daniels, B., Lord, S. J., Kiely, B. E., Houssami, N., Haywood, P., Lu, C. Y. et al. Use and outcomes of targeted therapies in early and metastatic HER2-positive breast cancer in Australia: protocol detailing observations in a whole of population cohort. BMJ Open 7, e014439 (2017).

37. Srasuebkul, P., Dobbins, T. A. \& Pearson, S. A. Validation of a proxy for estrogen receptor status in breast cancer patients using dispensing data. Asia Pac. J. Clin. Oncol. 10, e63-e68 (2014).

38. Suissa, S. Immortal time bias in pharmacoepidemiology. Am. J. Epidemiol. 167, 492-499 (2007).

39. Sateren, W. B., Trimble, E. L., Abrams, J., Brawley, O., Breen, N., Ford, L. et al. How sociodemographics, presence of oncology specialists, and hospital cancer programs affect accrual to cancer treatment trials. J. Clin. Oncol. 20, 2109-2117 (2002).

40. Gross, C. P., Filardo, G., Mayne, S. T. \& Krumholz, H. M. The impact of socioeconomic status and race on trial participation for older women with breast cancer. Cancer 103, 483-491 (2005).

41. Arpino, G., Michelotti, A., Truini, M., Montemurro, F., Russo, S., Palumbo, R. et al. Demographic, tumor and clinical features of clinical trials versus clinical practice patients with HER2-positive early breast cancer: results of a prospective study. J. Cancer Res. Clin. Oncol. 142, 669-678 (2016).

42. Seferina, S. C., Lobbezoo, D. J., de Boer, M., Dercksen, M. W., van den Berkmortel, F., van Kampen, R. J. et al. Real-life use and effectiveness of adjuvant trastuzumab in early breast cancer patients: a study of the Southeast Netherlands Breast Cancer Consortium. Oncologist 20, 856-863 (2015). 
Treatment patterns and survival in HER2-positive early breast cancer: a... $M$ Tang et al.

43. Chen L., Zhou W., Hu X., Yi M., Ye C. \& Yao G. Short-duration versus 1-year adjuvant trastuzumab in early HER2 positive breast cancer: A meta-analysis of randomized controlled trials. Cancer Treat. Rev. 75, 12-19 (2019).

44. Perez, E. A., Romond, E. H., Suman, V. J., Jeong, J.-H., Davidson, N. E., Geyer, C. E. Jr et al. Four-year follow-up of trastuzumab plus adjuvant chemotherapy for operable human epidermal growth factor receptor 2-positive breast cancer: joint analysis of data from NCCTG N9831 and NSABP B-31. J. Clin. Oncol. 29, 3366 (2011).

45. Martin, M., Holmes, F. A., Ejlertsen, B., Delaloge, S., Moy, B., Iwata, H. et al. Neratinib after trastuzumab-based adjuvant therapy in HER2-positive breast cancer (ExteNET): 5-year analysis of a randomised, double-blind, placebo-controlled, phase 3 trial. Lancet Oncol. 18, 1688-1700 (2017). 